\title{
Screening of three-dimensional spheroids of ovarian cancer: identification of novel therapeutics targeting stemness and chemoresistance
}

\author{
Neveen Said \\ Departments of Cancer Biology, Pathology, and Urology, Wake Forest University School of Medicine and Wake Forest Baptist Comprehensive \\ Cancer Center, Winston Salem, NC, USA \\ Correspondence to: Neveen Said, MD, PhD. Departments of Cancer Biology, Pathology and Urology, Wake Forest University School of Medicine, \\ Medical Center Blvd, Winston Salem, NC 27157, USA. Email: nsaid@wakehealth.edu. \\ Comment on: Hirst J, Pathak HB, Hyter S, et al. Licofelone Enhances the Efficacy of Paclitaxel in Ovarian Cancer by Reversing Drug Resistance and \\ Tumor Stem-like Properties. Cancer Res 2018;78:4370-85.
}

Submitted Sep 08, 2018. Accepted for publication Sep 12, 2018.

doi: $10.21037 / \mathrm{atm} .2018 .09 .41$

View this article at: http://dx.doi.org/10.21037/atm.2018.09.41

Epithelial ovarian cancer (OvCa) is the leading cause of death from gynecologic malignancies in the United States (1). More than $75 \%$ of patients are diagnosed at late stages due to the insipient protracted nature of the disease and lack of specific diagnostic symptoms and/or biomarkers (2). Despite aggressive surgical debulking and cytoreduction, $80 \%$ patients experience recurrence of a chemo-resistant disease with limited treatment options $(1,3)$. The standard of care (SOC) treatment is aggressive surgery followed by platinum-taxane chemotherapy, or neo-adjuvant chemotherapy followed by surgery (2). However, cisplatinresistance and recurrence develop in $25 \%$ of patients as early as 6 months, with the overall 5 -year survival hovering around $30-40 \%(1,2)$.

Despite recent advances in developing chemotherapeutics, treatment options for patients with ovarian cancer are still limited. This limitation is attributed in part to initial testing and validation of cancer cells grown in $2 \mathrm{D}$ monolayers in tissue culture plates that do not faithfully recapitulate the human disease as these cells are presented with an artificial growth surface (plastic) and a 2D architecture instead of 3D normal tissue. These conditions impose a selective pressure on cells, which could substantially alter their phenotype and molecular properties and would provide misleading and non-predictive data for in vivo responses $(4,5)$. Therefore, there is unmet need for development of novel preclinical models for evaluation of therapeutics that target OvCa cells and faithfully recapitulate the in vivo milieu. As a consequence, few compounds ( $10 \%)$ progress to clinical development and many of them fail during clinical trials, due to either lack of clinical efficacy and/or unacceptable toxicity (6). To overcome these limitations, and bridge the critical gap between the need for predictive in vitro platforms, and the availability of bio-tools and techniques, a recent study by Hirst and colleagues (7) from Dr. Andrew K. Godwin group developed a novel approach for drug discovery based on screening their activity in $3 \mathrm{D}$ multicellular tumor spheroids (MCTSs) to identify novel candidates, that are often missed by traditional screening in $2 \mathrm{D}$ cultures.

They reported that growing ovarian cancer cell lines in MCTSs phenocopied the characteristics of the in vivo tumors with a gradient of Ki67-positive cells being highest at the surface and negative in the center as well as a central hypoxic core. Hypoxia-inducible factor- $\alpha$ (HIF $1 \alpha)$ was stabilized in spheroids under normoxic conditions. In contrast, cancer cells grown in $2 \mathrm{D}$ cultures exhibited ubiquitous Ki67-staining and HIF1 $\alpha$ was stabilization only under hypoxic conditions. Consistently, hypoxia-regulated genes and markers of cancer stem cells (CSCs) and well as canonical stem cell markers were significantly increased in MCTSs relative to their 2D-cultures. MCTSs exhibited a robust paclitaxel-resistance with upregulation of hypoxiaregulated stem cell markers and decreased proliferation following paclitaxel treatment compared with 2D cultures. Investigators confirmed that paclitaxel-resistance and 
stemness are not driven by reduced drug penetration, but are rather due to stable phenotypic changes in ovarian cancer cells in 3D MCTS. They presented evidence that paclitaxel-resistance, and increased markers of stemness persisted after dissociation of cells from MCTSs and growing them in 2D culture. Furthermore, they showed that drugs with limited clinical activity despite their robust in vitro activity in 2D-cultures as dasatinib, nilotinib, and ganetespib, induced extensive expression of stem-like genes in 3D MCTSs. The investigators then concluded that the 3D MCTSs induces a chemo-resistant stem-like phenotype that warrants their use for first-line drug screening.

The authors carried further their hypothesis to identify FDA-approved drugs that can be repurposed to accelerate a path to the clinic. They developed a novel approach integrating RNASeq with in silico analysis to identify the regulatory pathways enriched in $3 \mathrm{D}$ MCTSs compared to 2D. With stringent cutoffs of top up- and down-regulated genes and in silico pathway prediction, they discovered that the top upstream regulators were related to hypoxia and the top drug hits were a nonsteroidal anti-inflammatory drugs (NSAID), fluticasone, and an antidiabetic, rosiglitazone. Thus, they constructed a focused drug library that included known cancer therapeutics, anti-inflammatory and compounds that target metabolic disorders. They reported that common hits in $2 \mathrm{D}$ and $3 \mathrm{D}$ were predominantly from the current cancer therapeutics that would be identified and validated by traditional screening approaches. Interestingly, the top unique 3D two hits were NSAID drugs, licofelone and glafenine, that have not been evaluated in preclinical or clinical studies for EOC.

Licofelone is a dual inhibitor of cyclooxygenase-2 and 5-lipoxygenase (COX-2/5-LOX) that was initially developed for the treatment of arthritis (8), whereas, glafenine, is a broad spectrum pain killer and an NSAID derivative of anthranilic acid (9). Both drugs reversed the stem-like markers and properties of ovarian cells grown in 3D spheroids, and their effect was cell line-specific though independent of their mutational profile. The investigators selected licofelone for further investigation due its safer clinical profile compared to glafenine (9-13). They further demonstrated that the enhanced cytotoxic activity of licofelone in cells in 3D MCTSs correlated with their higher basal expression of CSCs marker ALHD1A. Because epidemiologic studies showed that the use of NSAIDs, specifically aspirin, lowered the risk of EOC in a dose- and frequency-dependent manner (14). The authors compared the activity of licofelone with another NSAID, celecoxib which is a selective COX-2 inhibitor, on MCTSs. They provided evidence that licofelone exhibits more potent cytotoxic activity than in MCTSs suggesting that dual targeting of COX/LOX have superior inhibitory effect on ovarian MCTSs than COX-2 inhibition alone. Moreover, licofelone exhibited a synergistic effect with paclitaxel on $3 \mathrm{D}$ spheroids. Importantly, combinatorial treatment of licofelone and paclitaxel led to a significant reduction in the expression of a number of stem-like transcripts in MCTSs and could even reverse the stem-like phenotype induced by paclitaxel treatment.

Indeed, ovarian CSCs have been implicated as a cause of chemoresistance and recurrence (15-17). Studies of matching primary and recurrent chemoresistant ovarian tumors revealed that primary tumors are enriched with CSCs and stem cell pathway mediators, especially at the completion of primary therapy (17-19).

Ovarian CSCs have been shown to exhibit distinctive metabolic phenotype as they favor oxidative phosphorylation (OXPHOS) over glucose metabolism, and shunt pyruvate to the Krebs cycle, increasing reactive oxygen species (ROS) production and inducing hypoxia-resistant metabolism $(15,18,20,21)$. Recent studies have also shown that mitochondrial metabolism is critical for cancer stem-like cell phenotype $(16,18)$. Targeting mitochondrial function by inhibiting complex I and III showed a reduction in OXPHOS and inhibited ovarian CSCs $(21,22)$. In addition, licofelone has been shown to induce mitochondrial apoptosis in colon cancer cells independent of arachidonic acid inhibition (23). In this respect, Hirst and colleagues (7) showed that licofelone strongly downregulated paclitaxelinduced TCA-related genes and reduced the expression of multiple OXPHOS complexes (I, II, and IV). The effect was significantly robust in OVCAR8 spheroids that express high basal levels of stem cell markers, whereas no noticeable effect was observed in A1847 spheroids that express low basal levels of stem cell markers. The authors inferred that licofelone could be modulating the expression of stem-like markers through regulation of mitochondrial metabolism and OXPHOS.

The authors furthered their studies in vivo to demonstrate the efficacy of licofelone in an ovarian cancer PDX ascites model. Licofelone treatment showed minimal difference in median survival of mice as a single agent. However, in the combination therapy with paclitaxel, the median survival was significantly improved compared with vehicle alone or mono-therapy with either drug. Paclitaxel treatment significantly increased the expression 
of transcripts of stemness markers $C D 133$ and $A L D H 1 A$, whereas the combination therapy of licofelone and paclitaxel significantly reversed the expression of $A L D H 1 A$ compared with paclitaxel alone. Interestingly, the percent of $\mathrm{Ki}-67$-positive cells in tumor cells isolated from the ascites was significantly reduced in the paclitaxel-treated group compared with vehicle. However, in the licofelone-treated tumor cells, Ki-67-positive cells were significantly increased compared with vehicle, while the combination of paclitaxel and licofelone showed no statistical different compared with vehicle. Taken together these data further supported the observations that licofelone reverses paclitaxel-induced stemness and increases post-chemotherapy proliferative cells that can be targeted by SOC therapy.

In summary, Hirst and colleagues (7) provided a strong evidence that $3 \mathrm{D}$ in vitro screening is superior to traditional screening approaches for identifying new drug leads for the treatment of women with chemotherapy-refractory ovarian cancer, and perhaps more robust drug leads for other solid tumors. They showed that some molecular targeted agents with limited clinical efficacy induce stem-like phenotype after high-dose treatment.

\section{Acknowledgements}

Funding: This work was supported by Marsha Rivkin Pilot award, Wake Forest University Department of Pathology Endowment Funds and R01 CA193437 to N Said.

\section{Footnote}

Conflicts of Interest: The author has no conflicts of interest to declare.

\section{References}

1. Siegel RL, Miller KD, Jemal A. Cancer statistics, 2018. CA Cancer J Clin 2018;68:7-30.

2. Rizzuto I, Stavraka C, Chatterjee J, et al. Risk of Ovarian Cancer Relapse score: a prognostic algorithm to predict relapse following treatment for advanced ovarian cancer. Int J Gynecol Cancer 2015;25:416-22.

3. Borley J, Wilhelm-Benartzi C, Brown R, et al. Does tumour biology determine surgical success in the treatment of epithelial ovarian cancer? A systematic literature review. Br J Cancer 2012;107:1069-74.

4. Breslin S, O'Driscoll L. Three-dimensional cell culture: the missing link in drug discovery. Drug Discov Today
2013;18:240-9.

5. Torsvik A, Stieber D, Enger PØ, et al. U-251 revisited: genetic drift and phenotypic consequences of long-term cultures of glioblastoma cells. Cancer Med 2014;3:812-24.

6. Hopkins AL. Network pharmacology: the next paradigm in drug discovery. Nat Chem Biol 2008;4:682.

7. Hirst J, Pathak HB, Hyter S, et al. Licofelone Enhances the Efficacy of Paclitaxel in Ovarian Cancer by Reversing Drug Resistance and Tumor Stem-like Properties. Cancer Res 2018;78:4370-85.

8. Bias P, Buchner A, Klesser B, et al. The Gastrointestinal Tolerability of the LOX/COX Inhibitor, Licofelone, is Similar to Placebo and Superior to Naproxen Therapy in Healthy Volunteers: Results From a Randomized, Controlled Trial. Am J Gastroenterol 2004;99:611.

9. Boeijinga JK, van der Vijgh WJ. Double blind study of the effect of glafenine (Glifanan) on oral anticoagulant therapy with phenprocoumon (Marcumar). Eur J Clin Pharmacol 1977;12:291-6.

10. Amor B, Benarrosh C. A method for comparing analgesics: glafenine and paracetamol. Multicenter cross-over approach. Clin Rheumatol 1988;7:492-7.

11. Cledes J, Kermanach P, Guillodo MP, et al. Acute kidney failure after ingestion of glafenine. The role of associated alcoholic absorption. Presse Med 1990;19:333.

12. Davido A, Hallali P, Boutchnei T. Shock caused by glafenine. Apropos of 7 complications. Rev Med Interne 1989;10:113-7.

13. Stricker BH, de Groot RR, Wilson JH. Anaphylaxis to glafenine. Lancet 1990;336:943-4.

14. Trabert B, Ness RB, Lo-Ciganic WH, et al. Aspirin, nonaspirin nonsteroidal anti-inflammatory drug, and acetaminophen use and risk of invasive epithelial ovarian cancer: a pooled analysis in the Ovarian Cancer Association Consortium. J Natl Cancer Inst 2014;106:djt431.

15. Liao J, Qian F, Tchabo N, et al. Ovarian Cancer Spheroid Cells with Stem Cell-Like Properties Contribute to Tumor Generation, Metastasis and Chemotherapy Resistance through Hypoxia-Resistant Metabolism. PLoS One 2014;9:e84941.

16. Sancho P, Barneda D, Heeschen C. Hallmarks of cancer stem cell metabolism. Br J Cancer 2016;114:1305-12.

17. Steg AD, Bevis KS, Katre AA, et al. Stem Cell Pathways Contribute to Clinical Chemoresistance in Ovarian Cancer. Clin Cancer Res 2012;18:869-81.

18. Pastò A, Bellio C, Pilotto G, et al. Cancer stem cells from epithelial ovarian cancer patients privilege oxidative phosphorylation, and resist glucose deprivation. 


\section{Page 4 of 4}

Oncotarget 2014;5:4305-19.

19. Raghavan S, Mehta P, Ward MR, et al. Personalized Medicine-Based Approach to Model Patterns of Chemoresistance and Tumor Recurrence Using Ovarian Cancer Stem Cell Spheroids. Clin Cancer Res 2017;23:6934-45.

20. Alvero AB, Montagna MK, Sumi NJ, et al. Multiple blocks in the engagement of oxidative phosphorylation in putative ovarian cancer stem cells: implication for maintenance therapy with glycolysis inhibitors. Oncotarget 2014;5:8703-15.

21. Fiorillo M, Lamb R, Tanowitz HB, et al. Repurposing

Cite this article as: Said N. Screening of three-dimensional spheroids of ovarian cancer: identification of novel therapeutics targeting stemness and chemoresistance. Ann Transl Med 2018;6(Suppl 1):S26. doi: 10.21037/atm.2018.09.41

\section{Said. Targeting ovarian cancer stemness and chemoresistance}

atovaquone: Targeting mitochondrial complex III and OXPHOS to eradicate cancer stem cells. Oncotarget 2016;7:34084-99.

22. Shank JJ, Yang K, Ghannam J, et al. Metformin targets ovarian cancer stem cells in vitro and in vivo. Gynecol Oncol 2012;127:390-7.

23. Tavolari S, Bonafè $M$, Marini $M$, et al. Licofelone, a dual COX/5-LOX inhibitor, induces apoptosis in HCA7 colon cancer cells through the mitochondrial pathway independently from its ability to affect the arachidonic acid cascade. Carcinog 2008;29:371-80. 